\title{
Efficient Closed-Loop Schemes for MIMO-OFDM-Based WLANs
}

\author{
Xiayu Zheng, ${ }^{1}$ Yi Jiang, ${ }^{2}$ and Jian $\mathrm{Li}^{1}$ \\ ${ }^{1}$ Department of Electrical and Computer Engineering, University of Florida, Gainesville, FL 32611-6130, USA \\ ${ }^{2}$ Department of Electrical and Computer Engineering, University of Colorado, Boulder, CO 80309-0425, USA
}

Received 28 December 2005; Revised 18 July 2006; Accepted 13 August 2006

\begin{abstract}
The single-input single-output (SISO) orthogonal frequency-division multiplexing (OFDM) systems for wireless local area networks (WLAN) defined by the IEEE 802.11a standard can support data rates up to $54 \mathrm{Mbps}$. In this paper, we consider deploying two transmit and two receive antennas to increase the data rate up to $108 \mathrm{Mbps}$. Applying our recent multiple-input multipleoutput (MIMO) transceiver designs, that is, the geometric mean decomposition (GMD) and the uniform channel decomposition (UCD) schemes, we propose simple and efficient closed-loop MIMO-OFDM designs for much improved performance, compared to the standard singular value decomposition (SVD) based schemes as well as the open-loop V-BLAST (vertical Bell Labs layered space-time) based counterparts. In the explicit feedback mode, precoder feedback is needed for the proposed schemes. We show that the overhead of feedback can be made very moderate by using a vector quantization method. In the time-division duplex (TDD) mode where the channel reciprocity is exploited, our schemes turn out to be robust against the mismatch between the uplink and downlink channels. The advantages of our schemes are demonstrated via extensive numerical examples.
\end{abstract}

Copyright (c) 2006 Xiayu Zheng et al. This is an open access article distributed under the Creative Commons Attribution License, which permits unrestricted use, distribution, and reproduction in any medium, provided the original work is properly cited.

\section{INTRODUCTION}

The single-input single-output (SISO) orthogonal frequency-division multiplexing (OFDM) systems for wireless local area networks (WLAN) defined by the IEEE 802.11a standard can support data rates up to $54 \mathrm{Mbps}$ [1]. Improving the data rate to over $100 \mathrm{Mbps}$ is a major goal of the next-generation WLANs $[2,3]$. The multiple-input multipleoutput (MIMO) communication technology is widely regarded as a key to achieve such a high data rate.

Assuming that the channel state information (CSI) is available at both the transmitter and the receiver, the MIMO channel can be decoupled, using singular value decomposition (SVD), into multiple orthogonal subchannels (or eigenmodes) on each subcarrier [4]. To maximize the channel throughout, power allocation and bit loading should be applied to the subchannels in both the spatial and frequency domains (see, e.g., [5] and the references therein). However, bit loading is often not adopted in practice, such as in the IEEE 802.11 standards, due to its complexity. If the same constellation is used across all the subchannels, the weaker eigenmodes corresponding to the smaller singular values of the channel matrices tend to experience deeper fading [4], which degrades the overall system performance significantly. In [6], a power allocation method was proposed based on the minimum mean-squared error (MMSE) criterion for the
MIMO systems. This method tends to put more power on the weaker subchannels, which may cause significant capacity loss.

In this paper we propose simple and efficient closed-loop designs for MIMO-OFDM-based WLANs. We focus on the IEEE 802.11a standard, although our schemes are also applicable to other standards including the US standard IEEE 802.11g and the European standard HIPERLAN/2 [7]. Our schemes combine the recently proposed geometric mean decomposition (GMD) and uniform channel decomposition (UCD) transceiver designs $[8,9]$ with horizontal encoding and successive (noniterative) decoding. (An idea similar to GMD appeared in the independent work of [10].) GMD and UCD decompose each MIMO channel into multiple equal gain subchannels for each subcarrier, which allows our designs to obviate the need of any power allocations. The simulation results show that our closed-loop schemes enjoy multi$\mathrm{dB}$ improvement compared to the standard singular value decomposition (SVD) based schemes as well as the openloop V-BLAST (vertical Bell labs layered space-time) based counterparts.

In the explicit feedback mode, precoder feedback is required for the proposed schemes. We present a vector quantization algorithm for efficient precoder quantization. This quantization algorithm is inspired by an observation of the interesting link between a $2 \times 2$ unitary matrix and a $2 \mathrm{D}$ 


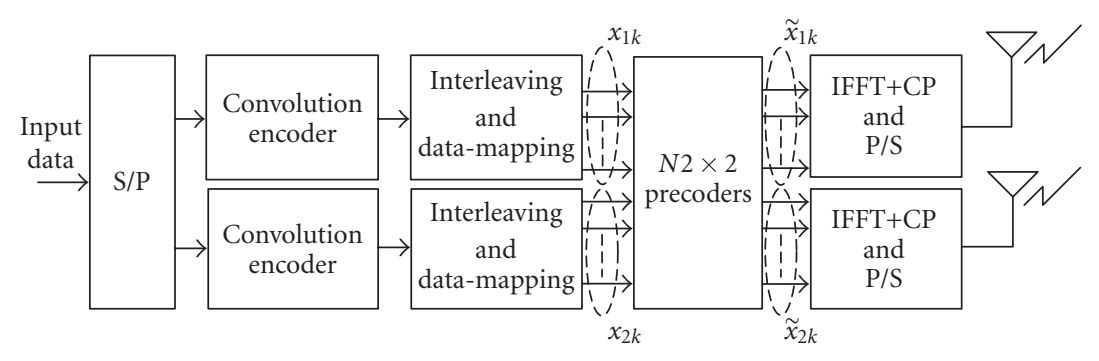

Figure 1: Transmitter design for MIMO-OFDM-based WLAN.

unit sphere. We show that the $2 \times 2$ unitary precoder matrix for each frequency subcarrier can be quantized by 6 bits with very small performance degradations. In the time-division duplex (TDD) mode, where the channel reciprocity principle holds [3], our schemes do not require any precoder feedback. With a simple modification, our schemes can be made quite robust against the uplink-downlink channel mismatches.

The remainder of this paper is organized as follows. Section 2 describes the $2 \times 2$ MIMO channel model with spatial correlations. Section 3 presents our closed-loop MIMO WLAN system configuration, including the precoder and equalizer designs, and the successive soft decoding approach. In Section 4, we consider the explicit feedback mode and provide two quantization methods for precoder feedback, where a new vector quantization algorithm is proposed. In Section 5, we consider the TDD mode and show that the proposed schemes can be made quite robust against the uplink-downlink channel mismatches. Numerical examples are given in Section 6 to demonstrate the effectiveness of our schemes. We conclude the paper in Section 7.

\section{Notation}

We use bold upper case letters to denote matrices and bold lower case letters for column vectors. We use $(\cdot)^{T}$ to denote the transpose and $(\cdot)^{*}$ to denote the Hermitian transpose. $\|\cdot\|$ stands for the Euclidean norm and $\|\cdot\|_{F}$ for the Frobenius norm. $\mathbf{I}_{N}$ is the $N \times N$ identity matrix; $\operatorname{det}(\cdot)$ is the determinant of a matrix and $E[\cdot]$ denotes the expectation operation.

\section{CHANNEL MODEL}

Consider a $2 \times 2$ MIMO channel with spatial correlations, where the channel can be modeled as [11]:

$$
\mathbf{h}(t)=\mathbf{R}_{r}^{1 / 2}\left[\begin{array}{ll}
h_{11}(t) & h_{12}(t) \\
h_{21}(t) & h_{22}(t)
\end{array}\right] \mathbf{R}_{t}^{1 / 2}
$$

with $\mathbf{R}_{t}$ and $\mathbf{R}_{r}$ quantifying the spatial correlations of the channel fading at the transmitter and the receiver, respectively, and

$$
h_{i j}(t)=\sum_{l=0}^{L-1} h_{i j}^{(l)} \delta\left(t-l T_{s}\right), \quad 1 \leq i, j \leq 2,
$$

denoting the frequency-selective channel link between the $j$ th transmit and $i$ th receive antennas (with $L$ being the channel length and $T_{s}$ the sampling period). The random variables $\left\{h_{i j}^{(l)}\right\}_{i, j=1}^{2}, l=1, \ldots, L$, are assumed to be independently distributed zero-mean, circularly symmetric complex Gaussian variables. Applying $N_{c}$-point fast Fourier transform (FFT) to $\mathbf{h}(t)$, we obtain the channel response in the frequency domain:

$$
\mathbf{H}(n)=\sum_{l=0}^{L-1} \mathbf{h}\left(l T_{s}\right) e^{-j 2 \pi n l / N_{c}}, \quad 1 \leq n \leq N_{c} .
$$

We denote by

$$
\mathbf{H}_{k} \triangleq \mathbf{H}(f(k))=\left[\begin{array}{ll}
H_{11}(f(k)) & H_{12}(f(k)) \\
H_{21}(f(k)) & H_{22}(f(k))
\end{array}\right], \quad 1 \leq k \leq N
$$

the flat fading channel matrix at the $k$ th data subcarrier $\left(N_{c}=64\right.$ and $N=48$ for IEEE 802.11a), with $f(k), 1 \leq k \leq$ $N$, denoting the data subcarrier mapping function in [1].

\section{CLOSED-LOOP MIMO WLAN SYSTEM DESIGN}

\subsection{System description}

Our MIMO-OFDM transmitter scheme is shown in Figure 1. We adopt the horizontal encoding method [12], where the two parallel branches perform encoding, bit interleaving, and data mapping separately. Let $x_{i k}$ denote the $i$ th encoded data symbol, $i=1,2$, on the $k$ th subcarrier, and let $\mathbf{x}_{k}=$ $\left[\begin{array}{ll}x_{1 k} & x_{2 k}\end{array}\right]^{T}$. On each of the $N$ data subcarriers, the transmitter applies a $2 \times 2$ precoder matrix $\mathbf{P}_{k}$ to obtain $\widetilde{\mathbf{x}}_{k}=\mathbf{P}_{k} \mathbf{x}_{k}$, $1 \leq k \leq N$. Denote by $\tilde{x}_{i k}$ the $i$ th element of $\tilde{\mathbf{x}}_{k}$. Then $\tilde{x}_{i k}$ is the symbol to be transmitted in the $i$ th branch at the $k$ th subcarrier. Consequently each precoded branch is OFDM modulated using an $N_{c}$-point IFFT and is added with a cyclic prefix (CP) before transmission. The length of CP is assumed to be longer than the channel length $L$, and therefore the intersymbol interference (ISI) can be completely eliminated at the receiver side.

In the explicit feedback mode, the precoders $\left\{\mathbf{P}_{k}\right\}_{k=1}^{N}$ are calculated and quantized at the receiver, and then fed back from the receiver to the transmitter. In the TDD mode, where the channel reciprocity principle holds, once the transmitter 


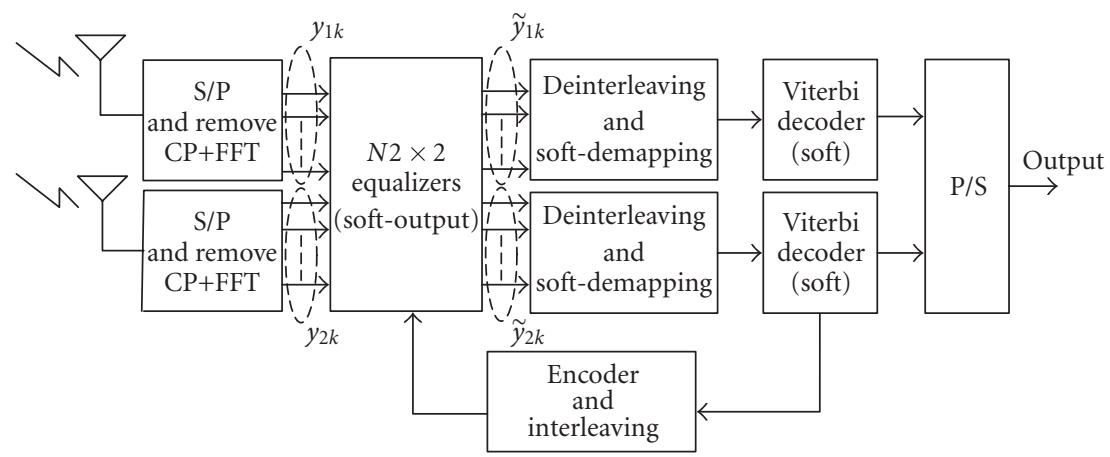

FIGURE 2: Receiver design for MIMO-OFDM-based WLAN.

estimates the reverse channel, that is, the one from the receiver to the transmitter, via training pilots, it can calculate the precoders $\mathbf{P}_{k}, k=1, \ldots, N$, to be used in the forward channel, that is, from the transmitter to the receiver.

Assuming accurate synchronization, frequency offset estimation and channel estimation, the receiver first removes the CP and applies an $N_{c}$-point FFT to each received branch as shown in Figure 2. Then the received signal vector at the $k$ th data subcarrier is

$$
\mathbf{y}_{k}=\mathbf{H}_{k} \mathbf{P}_{k} \mathbf{x}_{k}+\mathbf{z}_{k}, \quad k=1,2, \ldots, N
$$

where $\mathbf{z}_{k} \sim N\left(0, \sigma_{z}^{2} \mathbf{I}\right)$ denotes the circularly symmetric complex Gaussian noise. The key components of our closed-loop designs are the precoder $\mathbf{P}_{k}$ at the transmitter and the corresponding equalizers at the receiver, as we describe next.

\subsection{Precoder and equalizer design}

We design the precoder and equalizer based on our GMD and UCD transceiver design schemes $[8,9]$. Both schemes are based on the following theorem [8].

Theorem 1. Any rank $K$ matrix $\mathbf{H} \in \mathbb{C}^{M \times N}$ with singular values $\lambda_{H, 1} \geq \lambda_{H, 2} \geq \cdots \geq \lambda_{H, K}>0$ can be decomposed into

$$
\mathbf{H}=\mathbf{Q R P}^{*} \text {, }
$$

where $(\cdot)^{*}$ denotes the conjugate transpose, $\mathbf{R} \in \mathbb{R}^{K \times K}$ is an upper triangular matrix with equal diagonal elements $r_{i}=$ $\left(\prod_{n=1}^{K} \lambda_{H, n}\right)^{1 / K}, 1 \leq i \leq K$, and $\mathbf{Q} \in \mathbb{C}^{M \times K}$ and $\mathbf{P} \in \mathbb{C}^{N \times K}$ are semiunitary matrices.

Consider the channel model (5). In the explicit feedback mode, the GMD scheme [8] starts with the GMD matrix decomposition $\mathbf{H}_{k}=\mathbf{Q}_{k} \mathbf{R}_{k} \mathbf{P}_{k}^{*}$ at the receiver, to obtain $\mathbf{P}_{k}$, which is the unitary precoder to be fed back to the transmitter. Utilizing the precoder $\mathbf{P}_{k}$ at the transmitter as in (5) leads to the following received data vector:

$$
\mathbf{y}_{k}=\mathbf{Q}_{k} \mathbf{R}_{k} \mathbf{x}_{k}+\mathbf{z}_{k}, \quad k=1,2, \ldots, N
$$

At the receiver, multiplying $\mathbf{y}_{k}$ by $\mathbf{Q}_{k}^{*}$ yields

$$
\tilde{\mathbf{y}}_{k}=\mathbf{R}_{k} \mathbf{x}_{k}+\tilde{\mathbf{z}}_{k}
$$

where $\mathbf{R}_{k}=\mathbf{Q}_{k}^{*} \mathbf{H}_{k} \mathbf{P}_{k}$ is a $2 \times 2$ upper triangular matrix with equal diagonal and $\tilde{\mathbf{z}}_{k} \sim N\left(0, \sigma_{z}^{2} \mathbf{I}\right)$. The information symbols in $\mathbf{x}_{k}$ can then be detected successively starting from the second element of $x_{k}$ (see Section 3.3).

The UCD scheme [9] is somewhat more complicated than GMD. Like GMD, the UCD scheme has two implementations forms of which one can be regarded as a combination of a linear precoder with an MMSE V-BLAST equalizer. Compared to GMD, which suffers from capacity loss at lowto-moderate SNR, UCD is strictly capacity lossless and can achieve the optimal diversity-multiplexing gain tradeoff [13]. The details are omitted here due to limited space.

Both GMD and UCD obviate the need of bit loading and power allocation at the transmitter and require only the feedback of the unitary precoders $\mathbf{P}_{k}, k=1, \ldots, N$. In the TDD mode, the forward channel is estimated at the transmitter and therefore the precoders $\mathbf{P}_{k}$ can be calculated at the transmitter.

\subsection{Successive soft decoding}

Note that $\mathbf{R}_{k}$ is an upper triangular matrix. As shown in Figure 2, we adopt the schemes of deinterleaving, softdemapping and the low-complexity soft Viterbi decoder used in [2] for each branch separately. We first detect the data sequence of the lower branch to get the soft information. Assuming successful decoding of the data of the lower branch, we can cancel the interference due to the lower branch completely before decoding the upper branch, as is denoted by the feedback link at the lower part of Figure 2. The interference cancelation process of each subcarrier $k$ using GMD is outlined as follows.

\section{(1) Initial stage}

Calculate

$$
\begin{aligned}
& \sigma_{2 k}^{2}=E\left[\left|\frac{\tilde{z}_{2 k}}{\left(\mathbf{R}_{k}\right)_{22}}\right|^{2}\right]=\frac{\sigma_{z}^{2}}{\left(\mathbf{R}_{k}\right)_{22}^{2}}, \\
& \hat{x}_{2 k}=\frac{\tilde{y}_{2 k}}{\left(\mathbf{R}_{k}\right)_{22}}, \quad k=1, \ldots, N,
\end{aligned}
$$

where $\left(\mathbf{R}_{k}\right)_{i j}, i, j=1,2$, is the $(i, j)$ th element of $\mathbf{R}_{k}$ and $\tilde{y}_{2 k}$ is 
the second entry of $\tilde{\mathbf{y}}_{k}$. Note that $\sigma_{2 k}^{2}$ along with $\hat{x}_{2 k}$ provides the soft information for the lower branch. We can decode the lower branch data sequence by using the soft Viterbi decoder.

\section{(2) Cancellation stage}

Calculate

$$
\begin{aligned}
& \sigma_{1 k}^{2}=E\left[\left|\frac{\tilde{z}_{1 k}}{\left(\mathbf{R}_{k}\right)_{11}}\right|^{2}\right]=\frac{\sigma_{z}^{2}}{\left(\mathbf{R}_{k}\right)_{11}^{2}}=\sigma_{2 k}^{2}, \\
& \hat{x}_{1 k}=\frac{\left[\tilde{y}_{1 k}-\left(\mathbf{R}_{k}\right)_{12} \bar{x}_{2 k}\right]}{\left(\mathbf{R}_{k}\right)_{11}}, \quad k=1, \ldots, N,
\end{aligned}
$$

where $\sigma_{1 k}^{2}$ along with $\hat{x}_{1 k}$ provides the soft information for the upper branch. Here $\bar{x}_{2 k}$ is the reconstructed data symbol sequence obtained from the Viterbi-decoder of the lower branch. Note that $\sigma_{1 k}^{2}=\sigma_{2 k}^{2}$ because $\mathbf{R}_{k}$ has equal diagonal. Given the soft information for the upper branch, we can also decode the upper branch data sequence by using the soft Viterbi decoder.

For UCD, the successive soft decoding procedure is similar. Because $\sigma_{1 k}^{2}=\sigma_{2 k}^{2}$, the two branches have effectively the same output SNR. In contrast, the SVD-based or the conventional V-BLAST-based methods lead to two subchannels with unbalanced gains. For the systems with a fixed symbol constellation across all the subchannels, the weaker subchannel dominates the overall packet-error-rate (PER) performance, although iterative decoding between the two branches is helpful for reducing the PER of V-BLAST [12].

\section{PRECODER QUANTIZATION}

In the explicit feedback mode, the channel is estimated at the receiver. We compute the precoders $\mathbf{P}_{k}, k=1, \ldots, N$, at the receiver and feed them back to the transmitter. In the following, we present two quantization approaches to reduce the overhead of precoder feedback.

\subsection{Scalar quantization}

A simple scalar quantization scheme is as follows. Note that a $2 \times 2$ unitary precoder can be represented by

$\mathbf{P}(\theta, \phi)=\left[\begin{array}{cc}\cos \theta & -\sin \theta e^{-j \phi} \\ \sin \theta e^{j \phi} & \cos \theta\end{array}\right], \quad 0 \leq \theta<\pi, 0 \leq \phi<2 \pi$.

Denote

$$
\mathbf{P}\left(\theta_{n_{1}}, \phi_{n_{2}}\right)=\left[\begin{array}{cc}
\cos \theta_{n_{1}} & -\sin \theta_{n_{1}} e^{-j \phi_{n_{2}}} \\
\sin \theta_{n_{1}} e^{j \phi_{n_{2}}} & \cos \theta_{n_{1}}
\end{array}\right],
$$

where $\theta_{n_{1}}=\pi n_{1} / N_{1}, 0 \leq n_{1} \leq N_{1}-1, \phi_{n_{2}}=2 \pi n_{2} / N_{2}$, $0 \leq n_{2} \leq N_{2}-1$, with $N_{1}$ and $N_{2}$ denoting the quantization levels of $\theta_{n_{1}}$ and $\phi_{n_{2}}$, respectively. After obtaining the precoder $\mathbf{P}_{k}$ using GMD or UCD, we quantize $\mathbf{P}_{k}$ to the "closest" (via round off) grid point in (12). Hence for each subcarrier $k$, we only need to feed the index $\left(n_{1}, n_{2}\right)$ back to the transmitter, which requires $\log _{2}\left(N_{1} N_{2}\right)$ bits. To reduce the effect of quantization error and improve the robustness for GMD, instead of applying the original equalizer $\mathbf{Q}_{k}^{*}$ at the receiver, we instead use $\widetilde{\mathbf{Q}}_{k}^{*}$ obtained by the QR decomposition:

$$
\mathbf{H}_{k} \mathbf{P}\left(\theta_{n_{1}}, \phi_{n_{2}}\right) \triangleq \widetilde{\mathbf{Q}}_{k} \widetilde{\mathbf{R}}_{k}, \quad k=1, \ldots, N
$$

Note that $\mathbf{P}\left(\theta_{n_{1}}, \phi_{n_{2}}\right)$ is known at the receiver. We also need to replace $\mathbf{R}_{k}$ by $\widetilde{\mathbf{R}}_{k}$ in our interference cancelation stage. Clearly, when $N_{1}$ and $N_{2}$ are reasonably large, $\widetilde{\mathbf{R}}_{k}$ is approximately equal to $\mathbf{R}_{k}$ and the two diagonal elements of $\widetilde{\mathbf{R}}_{k}$ are almost the same, that is, the gains of the two branches remain almost the same. However, larger $N_{1}$ and $N_{2}$ also mean more feedback overhead. In practice, we need to choose $N_{1}$ and $N_{2}$ to achieve a reasonable tradeoff between feedback overhead and performance.

Similarly, we can apply the MMSE V-BLAST algorithm [14] to $\mathbf{H}_{k} \mathbf{P}\left(\theta_{n_{1}}, \phi_{n_{2}}\right)$ to obtain the equalizer when using UCD.

\subsection{Vector quantization}

Vector quantization can be adopted to further reduce the overhead of precoder feedback. We present a geometric approach to perform vector quantization. Suppose we quantize the precoder $\mathbf{P}(\theta, \phi)$ to be $\mathbf{P}(\hat{\theta}, \hat{\phi})$, where $(\hat{\theta}, \hat{\phi})$ correspond to an element in a codebook known to both the transmitter and receiver. Instead of transmitting the desired data vector $\mathbf{P}(\theta, \phi) \mathbf{x}_{k}$ at the transmitter, where $\mathbf{x}_{k}$ is the encoded data vector, we transmit $\mathbf{P}(\hat{\theta}, \hat{\phi}) \mathbf{x}_{k}$. To optimize the quantization scheme, we minimize the following cost function:

$$
\begin{aligned}
d & =E\left[\left\|\mathbf{P}(\theta, \phi) \mathbf{x}_{k}-\mathbf{P}(\hat{\theta}, \hat{\phi}) \mathbf{x}_{k}\right\|^{2}\right] \\
& =E\left[\mathbf{x}_{k}^{*}[\mathbf{P}(\theta, \phi)-\mathbf{P}(\hat{\theta}, \hat{\phi})]^{*}[\mathbf{P}(\theta, \phi)-\mathbf{P}(\hat{\theta}, \hat{\phi})] \mathbf{x}_{k}\right],
\end{aligned}
$$

with respect to $\hat{\theta}$ and $\hat{\phi}$. This cost function measures the average distortion caused by the finite rate precoder quantization. Here the expectation is over $\mathbf{x}_{k}$. After some straightforward algebra, we obtain

$$
\begin{aligned}
& {[\mathbf{P}(\theta, \phi)-\mathbf{P}(\hat{\theta}, \hat{\phi})]^{*}[\mathbf{P}(\theta, \phi)-\mathbf{P}(\hat{\theta}, \hat{\phi})]} \\
& \quad=2 \mathbf{I}_{2}-2[\cos \theta \cos \hat{\theta}+\sin \theta \sin \hat{\theta} \cos (\phi-\hat{\phi})] \mathbf{I}_{2} .
\end{aligned}
$$

Because the value of $E\left[\left\|\mathbf{x}_{k}\right\|^{2}\right]$ does not affect our quantization problem, without loss of generality, let $E\left[\left\|\mathbf{x}_{k}\right\|^{2}\right]=1$. Then

$$
\begin{aligned}
d & =2-2[\cos \theta \cos \hat{\theta}+\sin \theta \sin \hat{\theta} \cos (\phi-\hat{\phi})] \\
& \triangleq 2-2 \zeta
\end{aligned}
$$

In the following, we give a geometric interpretation of $\zeta$. We note that there is a one-to-one and onto mapping from the unitary precoder set $\{\mathbf{P}(\theta, \phi): 0 \leq \theta<\pi, 0 \leq \phi<2 \pi\}$ 
to the $2 \mathrm{D}$ unit sphere $\left\{\mathbf{v} \in \mathbb{R}^{3}:\|\mathbf{v}\|=1\right\}$. Any point on the $2 \mathrm{D}$ unit sphere with angles $(\theta, \phi)$ can be represented in the Cartesian coordinate as $\mathbf{v}=\left[\begin{array}{lll}\cos \theta & \sin \theta \cos \phi & \sin \theta \sin \phi\end{array}\right]^{T}$, where the first element of $\mathbf{v}$ is the $(1,1)$-element of $\mathbf{P}(\theta, \phi)$ and the second and third elements of $\mathbf{v}$, respectively, are the real and imaginary parts of the $(2,1)$-element of $\mathbf{P}(\theta, \phi)$. Each $\mathbf{P}(\theta, \phi)$ corresponds to a point $\mathbf{v}$ on the $2 \mathrm{D}$ unit sphere. Similarly, any point on the $2 \mathrm{D}$ unit sphere with angles $(\hat{\theta}, \hat{\phi})$ can be represented by the Cartesian coordinate $\hat{\mathbf{v}}=$ $[\cos \hat{\theta} \sin \hat{\theta} \cos \hat{\phi} \sin \hat{\theta} \sin \hat{\phi}]^{T}$. We see that $\zeta$ is just the inner product between $\mathbf{v}$ and $\hat{\mathbf{v}}$. Define $\psi$ as the angle between $\mathbf{v}$ and $\hat{\mathbf{v}}$. Then $\zeta=\cos \psi$ and

$$
d=(2-2 \cos \psi)=\|\mathbf{v}-\widehat{\mathbf{v}}\|^{2} .
$$

Based on this derivation, we conclude that a good codebook $\left\{\tilde{\mathbf{v}}_{i}\right\}_{i=1}^{N_{v}}$ should be distributed on the unit sphere as uniform as possible.

We use the following steps to determine the codebook. First, we generate a training set $\left\{\mathbf{v}_{n}, n=1,2, \ldots, N_{t}\right\}$ via randomly picking $N_{t}$ points on the $2 \mathrm{D}$ unit sphere, where $N_{t}$ is a very large number. Next, starting with an initial codebook (obtained via the splitting method [15]), we iteratively update the codebook [15] until no further improvement on the minimum distance is observed based on the following criteria.

(1) Nearest neighbor condition (NNC): for a given codebook $\left\{\widetilde{\mathbf{v}}_{i}\right\}_{i=1}^{N_{v}}$, assign a vector $\mathbf{v}_{n}$ to the $i$ th region

$$
S_{i}=\left\{\mathbf{v}_{n}:\left\|\mathbf{v}_{n}-\tilde{\mathbf{v}}_{i}\right\|^{2} \leq\left\|\mathbf{v}_{n}-\tilde{\mathbf{v}}_{j}\right\|^{2}, \forall j \neq i\right\},
$$

where $S_{i}, i=1,2, \ldots, N_{v}$, is the partition set for the $i$ th code vector.

(2) Centroid condition (CC): for a given partition $S_{i}$, the updated optimum code vectors $\left\{\tilde{\mathbf{v}}_{i}\right\}_{i=1}^{N_{v}}$ satisfy

$$
\tilde{\mathbf{v}}_{i}=\arg \min _{\left\|\widetilde{\mathbf{v}}_{i}\right\|=1} E\left[\left\|\mathbf{v}_{n}-\tilde{\mathbf{v}}_{i}\right\|^{2} \mid \mathbf{v}_{n} \in S_{i}\right], \quad i=1,2, \ldots, N_{v} .
$$

As shown in the appendix, the solution to the above optimization problem is

$$
\tilde{\mathbf{v}}_{i}=\frac{\overline{\mathbf{v}}_{i}}{\left\|\overline{\mathbf{v}}_{i}\right\|}, \quad i=1,2, \ldots, N_{v},
$$

where $\overline{\mathbf{v}}_{i}=\sum_{\mathbf{v}_{n} \in S_{i}} \mathbf{v}_{n} / \sum_{\mathbf{v}_{n} \in S_{i}} 1$ is the mean vector for the partition set $S_{i}, i=1,2, \ldots, N_{v}$.

Hence, for each subcarrier $k$, we first map the precoder $\mathbf{P}_{k}$ as a point $\mathbf{v}$ on the $2 \mathrm{D}$ unit sphere. According to the NNC criterion, we obtain the quantized vector $\hat{\mathbf{v}}$ from the codebook with index $i$. The index $i$ is fed back to the transmitter to reconstruct the precoder $\mathbf{P}(\hat{\theta}, \hat{\phi})$. In this case the overhead of feedback is $\log _{2}\left(N_{v}\right)$ bits per subcarrier.

\section{ROBUST TRANSCEIVER DESIGN IN THE TDD MODE}

In the TDD mode, the channel reciprocity can be exploited to obviate the need of precoder feedback in high throughput MIMO WLAN system [3]. However, there is always a mismatch between the forward channel (from transmitter to receiver) and reverse channel (from receiver to transmitter) due to channel variations and/or amplifier mismatches, which poses major difficulties of utilizing the conventional closed-loop schemes [16].

Our closed-loop schemes can be modified to be robust against the mismatches and be backward compatible with the standard open-loop V-BLAST receiver [8]. Denote by $\tilde{\mathbf{H}}_{k}$ the forward channel assumed by the transmitter and by $\mathbf{H}_{k}$ the actual channel matrix at the $k$ th data subcarrier. We may denote the channel mismatch as follows:

$$
\tilde{\mathbf{H}}_{k}=\mathbf{H}_{k}+\alpha \mathbf{E}, \quad 1 \leq k \leq N,
$$

where $\mathbf{E}$ is a matrix whose elements are independently and identically distributed (iid) complex-valued Gaussian variables with zero-mean and variance $\sigma^{2}=E\left[\left\|\mathbf{H}_{k}\right\|_{F}^{2}\right] / 4$, and $\alpha$ determines the level of channel mismatch. At the transmitter, the precoders $\widetilde{\mathbf{P}}_{k}, k=1, \ldots, N$, are obtained based on $\widetilde{\mathbf{H}}_{k}$, $k=1, \ldots, N$. The pilot (for channel estimation) and data sequences are both precoded using precoders $\widetilde{\mathbf{P}}_{k}, k=1, \ldots, N$, before transmission, which leads to the following received signals instead of (7):

$$
\mathbf{y}_{k}=\mathbf{H}_{k} \widetilde{\mathbf{P}}_{k} \mathbf{x}_{k}+\mathbf{z}_{k}, \quad k=1,2, \ldots, N .
$$

Assuming perfect channel estimation at the receiver, the estimated channel matrix on the $k$ th data subcarrier is the "virtual channel" $\mathbf{H}_{k} \widetilde{\mathbf{P}}_{k}$. As in Figure 2 , an equalizer $\overline{\mathbf{Q}}_{k}^{*}$ is applied to the $k$ th subcarrier to yield

$$
\tilde{\mathbf{y}}_{k}=\overline{\mathbf{Q}}_{k}^{*}\left(\mathbf{H}_{k} \widetilde{\mathbf{P}}_{k} \mathbf{x}_{k}+\mathbf{z}_{k}\right),
$$

where the equalizer $\overline{\mathbf{Q}}_{k}^{*}$ is obtained from the QR decomposition of $\mathbf{H}_{k} \widetilde{\mathbf{P}}_{k}$, that is, $\mathbf{H}_{k} \widetilde{\mathbf{P}}_{k}=\overline{\mathbf{Q}}_{k} \overline{\mathbf{R}}_{k}$. Hence

$$
\tilde{\mathbf{y}}_{k}=\overline{\mathbf{R}}_{k} \mathbf{x}_{k}+\tilde{\mathbf{z}}_{k}
$$

and we can apply successive soft decoding as described in Section 3.3 to retrieve the transmitted data on the $k$ th data subcarrier. Note that the channel gains of the two branches are usually unbalanced due to the mismatches between $\tilde{\mathbf{H}}_{k}$ and $\mathbf{H}_{k}$. However, for some small $\alpha$, the output SNRs of the two branches should be close, which results in only marginal performance loss, as shown with numerical examples in Section 6.

Similarly, for UCD, the precoder $\widetilde{\mathbf{P}}_{k}$ is calculated according to the UCD procedure based on $\widetilde{\mathbf{H}}_{k}$ and the receiver involves an MMSE V-BLAST equalizer.

\section{NUMERICAL EXAMPLES}

We present several numerical examples to demonstrate the superior performance of the proposed schemes. The system parameters used here are based on the IEEE 802.11a standard. For the two transmit and two receive antenna systems, the 64-QAM modulation and the channel coding rate of $R=3 / 4$ are used. The total frequency bandwidth is $20 \mathrm{MHz}$, which are divided into 64 subcarriers, including 48 data subcarriers. For each OFDM symbol with length 64 there is CP 
with length 16 which are discarded at the receiver to remove ISI. Therefore the total data rate is $2 \times \log _{2} 64 \times 3 / 4 \times 48 / 64 \times$ $20 \times 64 /(64+16)=108$ Mbps. The channel between each transmit and receive antenna pair is generated according to the Chayat model [17] with 50 ns root-mean-squared (RMS) delay spread (here the sampling period is $T_{s}=50 \mathrm{~ns}$ ). We assume that the channels are perfectly estimated at the receiver. The data are formatted into packets consisting of 1000 information bytes. According to IEEE 802.11a, the goal is to achieve the packet-error-rate (PER) of 0.1.

For the purpose of comparison, we also implement the following three standard schemes.

The first is a simple SVD-based scheme. For this scheme, both the transmitter and receiver apply unitary rotations to diagonalize the channel matrix at each subcarrier, which yields $2 \times 48=96$ orthogonal data subchannels. No bit allocation is involved here, since otherwise 256-QAM or larger constellations would be used, which would pose difficulties in the hardware implementations due to the phase noise issues, and so forth. The input power is uniformly allocated to all the 96 data subchannels. One encoder/decoder is sufficient in this case because the SVD completely eliminates the interference between subchannels and no successive decoding is needed.

The second scheme is similar to the first, except that the power allocation (PA) algorithm of [6] is applied at each subcarrier. Because the two subchannels at each subcarrier is usually highly unbalanced, this power allocation algorithm tends to compensate the weaker one with more power.

The third is an open-loop MMSE V-BLAST-based scheme. Just like the proposed GMD and UCD schemes, it applies two independent encoders and decoders for successive interference cancelation. Of course, unlike GMD and UCD, the two data branches have usually unbalanced channel gains. Improvement can be achieved via iterative decoding as described in the following. After decoding the lower branch, we can decode the upper branch with the influence from the lower branch canceled. Now given the decoded data from the upper branch, we can obtain improved decoding of the lower branch by removing the influence of the data from the upper branch. This procedure can be iterated.

We also include the channel outage probability curve as a benchmark. Channel outage probability is defined as the probability that the instantaneous mutual information of the channel,

$$
\begin{aligned}
I(\mathrm{SNR})= & 20 \times \frac{48}{64} \times \frac{64}{64+16} \\
& \times \sum_{k=1}^{48} \log \left[\operatorname{det}\left(\mathbf{I}_{2}+\frac{\mathrm{SNR}}{2} \mathbf{H}_{k} \mathbf{H}_{k}^{*}\right)\right],
\end{aligned}
$$

is less than 108. The channel outage probability is the lower bound of the PER performance of any MIMO scheme. An information-theoretically optimal scheme combined with a capacity-achieving code should be able to achieve this curve.

First, we consider channels without spatial correlation, that is, $\mathbf{R}_{r}=\mathbf{I}_{2}$ and $\mathbf{R}_{t}=\mathbf{I}_{2}$ (cf. (1)). Figure 3 shows the PER performances of the proposed GMD/UCD schemes, the closed-loop SVD with and without PA, and the open-loop

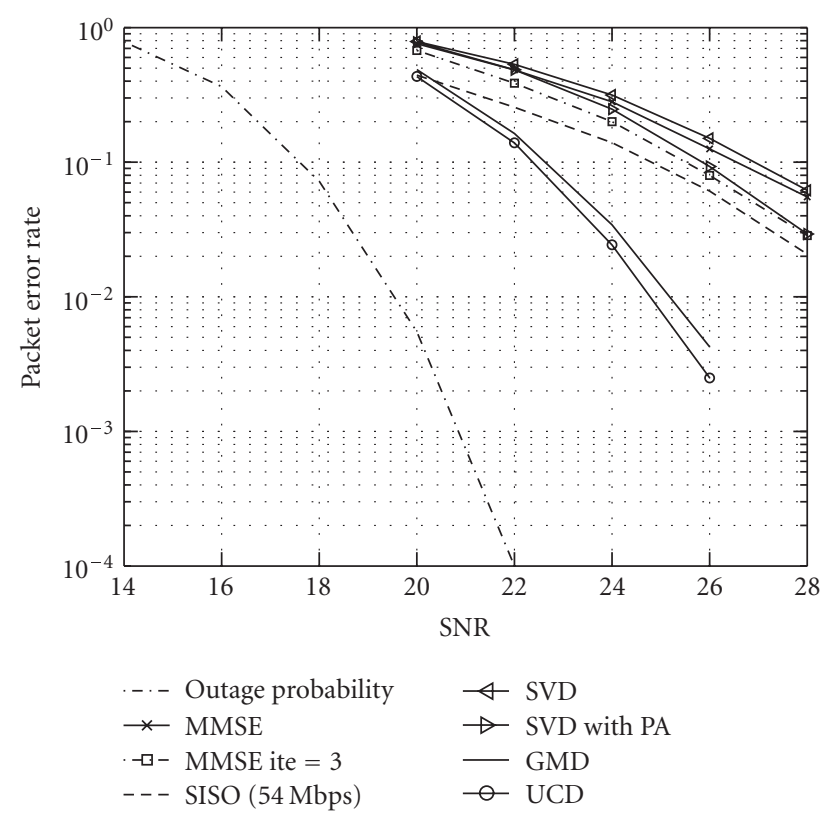

Figure 3: Performance comparison of MIMO WLAN (108 Mbps) schemes for uncorrelated channels in the absence of quantization errors.

MMSE V-BLAST [18] based scheme. We assume perfect precoder feedback. It can be seen from Figure 3 that the proposed closed-loop designs have more than $4 \mathrm{~dB}$ SNR improvement over the MMSE V-BLAST scheme at PER equal to 0.1 , although one can have a $1 \mathrm{~dB}$ gain by applying iterative decoding. The SVD-based method without PA has performance inferior to the open-loop MMSE V-BLAST-based scheme. The scheme based on SVD with PA performs better, but there is still more than $3 \mathrm{~dB}$ loss compared to the GMD and UCD schemes. The dashed line represents the performance of the $802.11 \mathrm{a}$ system with data rate $54 \mathrm{Mbps}$. It is remarkable that compared with the SISO scheme, our simple closed-loop $2 \times 2$ schemes can double the data rate and at the same time save $2.5 \mathrm{~dB}$ in total transmission power. Moreover, the PER curves of the GMD and UCD schemes have decreasing slopes much steeper than the other methods, which implies much improved diversity gain. There is still a gap of about $4.5 \mathrm{~dB}$ between the UCD scheme and the outage probability curve. Combined with a capacity achieving code, such as a Turbo code and a low density parity check code (LDPC) [19], the proposed schemes should close the gap further.

Figure 4 shows a typical example of the output SNRs of the eigen-subchannels $(-\diamond-$ and $-\square-)$ obtained by SVD at the 48 data subcarriers. We see that the weaker eigensubchannels have very low output SNR (say, less than $0 \mathrm{~dB}$ ). These weak subchannels may cause too many detection errors for the error control code to handle. However, at each subcarrier, the GMD and UCD schemes decompose a MIMO channel into two identical subchannels. The output SNRs of the subchannels of GMD and UCD are also shown in Figure 4 . We can see that the quality of the subchannels of GMD and UCD are much more stable across the subcarriers. 


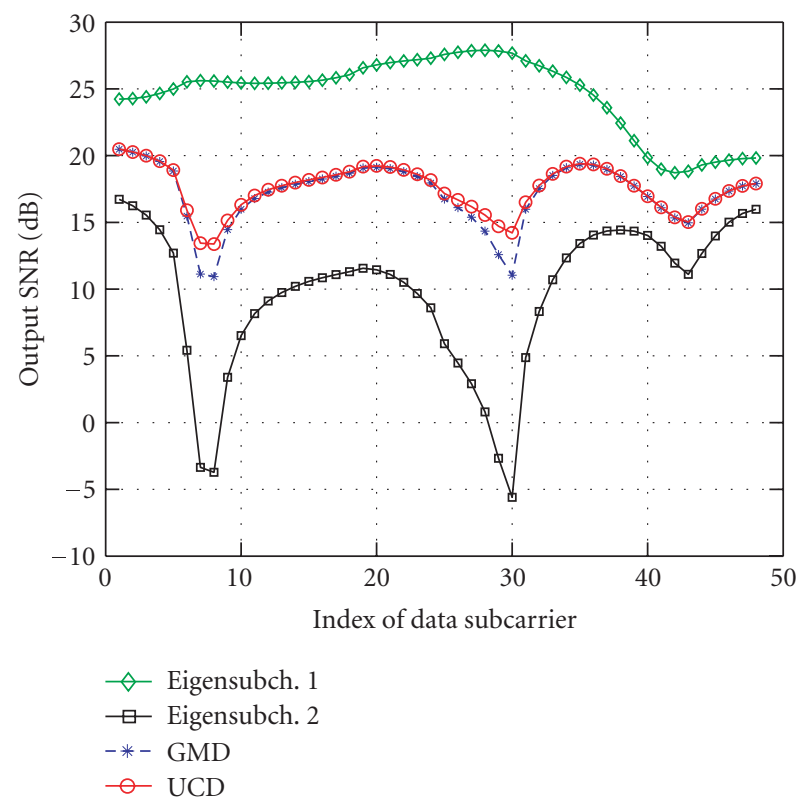

FIgURE 4: Output SNRs of the subchannels obtained via GMD, $\mathrm{UCD}$, and SVD, with input SNR $=22 \mathrm{~dB}$.

This figure provides insight into the reason why GMD and UCD perform significantly better than the SVD-based methods. We can also see that UCD outperforms GMD when the channel is close to singular, like the one at the 30th subcarrier.

In the second example, we consider a spatially correlated channel with

$$
\mathbf{R}_{r}=\left[\begin{array}{cc}
1 & 0.7 \\
0.7 & 1
\end{array}\right], \quad \mathbf{R}_{t}=\left[\begin{array}{cc}
1 & 0.3 \\
0.3 & 1
\end{array}\right]
$$

while all the other parameters remain the same as the first example. The results are given in Figure 5. Compared with Figure 3, in Figure 5 all the MIMO-OFDM schemes suffer from performance degradations due to the spatial fading correlations. However, the relative advantage of the proposed closed-loop schemes is even more prominent in this scenario. Specifically, the UCD scheme has a more than $4 \mathrm{~dB}$ gain over the SVD-based schemes and approximately a $6 \mathrm{~dB}$ gain over the open-loop MMSE V-BLAST scheme at PER equal to 0.1. Indeed, we expect that the eigen-subchannels obtained by SVD should have more disparate channel gains in the presence of fading correlations. Despite the fading correlations, the proposed GMD and UCD systems at the $108 \mathrm{Mbps}$ data rate still provide better PER performance than the SISO system at half the data rate.

We consider next the effect of quantized precoder on system performance. We use 8-bit scalar quantization with $N_{1}=2^{4}$ and $N_{2}=2^{4}$ (cf. Section 4.1) and $m$-bit vector quantization with $N_{v}=2^{m}, m=2,4,6$, (cf. Section 4.2) to quantize the precoder $\mathbf{P}_{k}$ of each data subcarrier. Figures 6 and 7 show that the 6-bit vector quantization performs equally well as the 8-bit scalar quantization. By using the 6-bit vec-

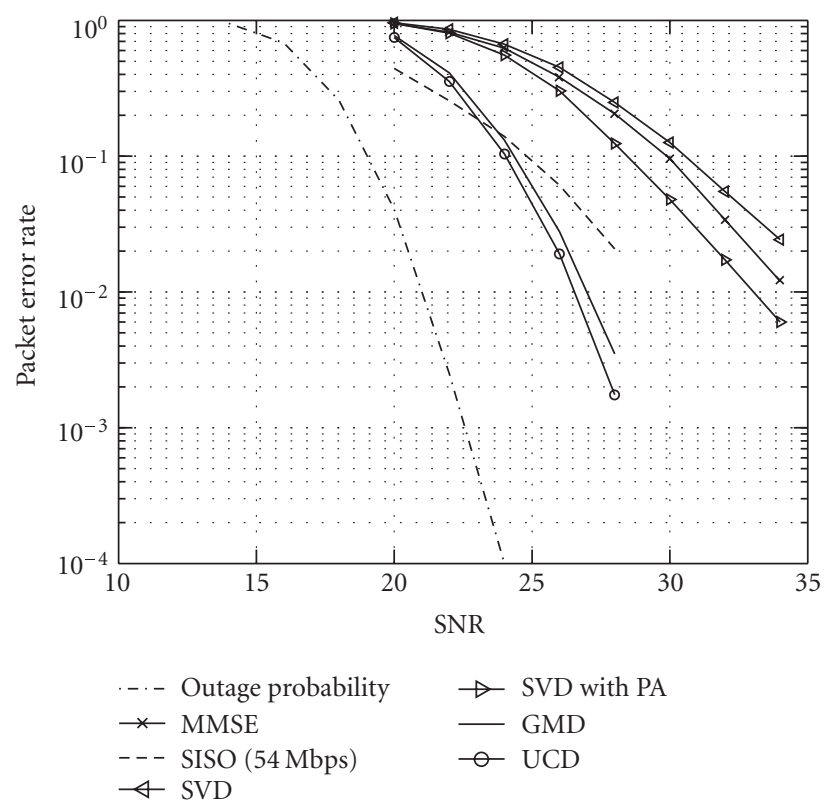

Figure 5: Performance comparison of MIMO WLAN (108 Mbps) schemes for correlated channels in the absence of quantization errors.

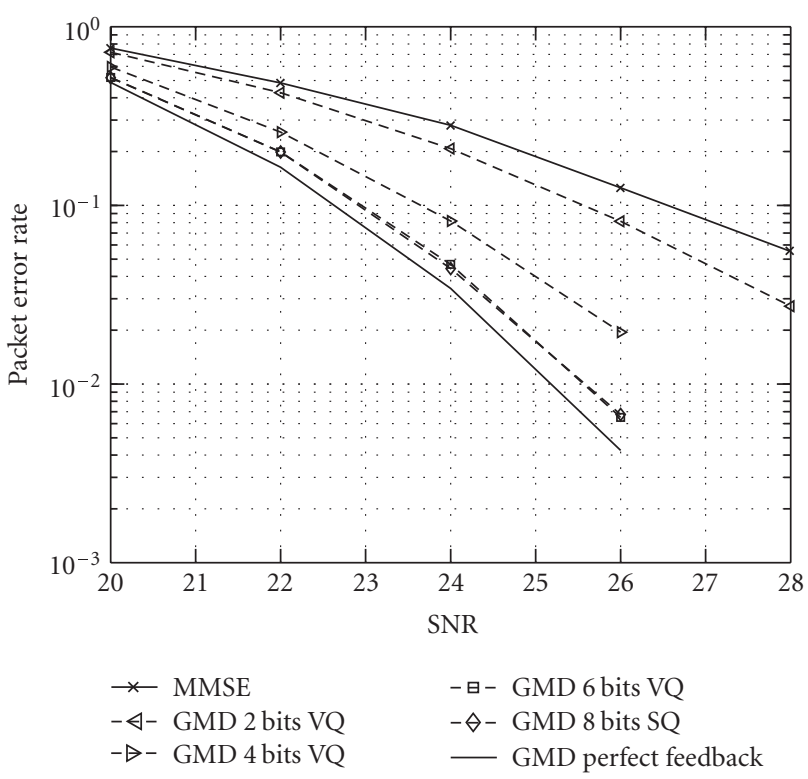

Figure 6: Performance comparison of the proposed closed-loop schemes for uncorrelated channels with 8-bit scalar quantization and various vector quantization bit rates per subcarrier in the explicit feedback mode for GMD.

tor quantization, our quantized closed-loop MIMO schemes suffer from less than $0.3 \mathrm{~dB}$ SNR loss compared to the perfect feedback case at PER $=0.1$. This small loss is negligible compared to the significant improvement of our proposed scheme over others. When more bits are used, we can further close the small gap. 


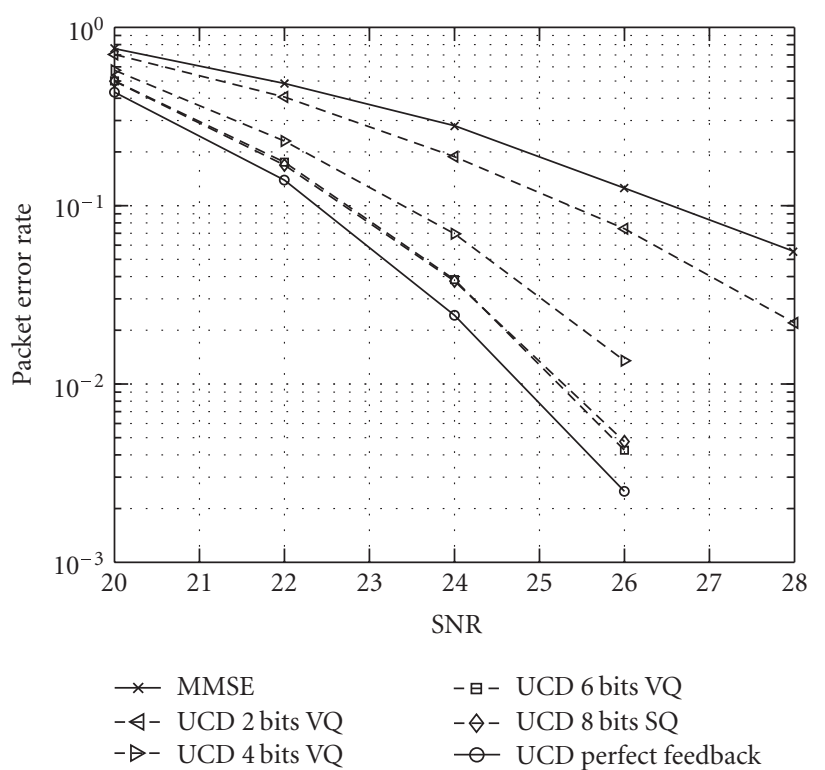

FIGURE 7: Performance comparison of the proposed closed-loop schemes for uncorrelated channels with 8-bit scalar quantization and various vector quantization bit rates per subcarrier in the explicit feedback mode for UCD.

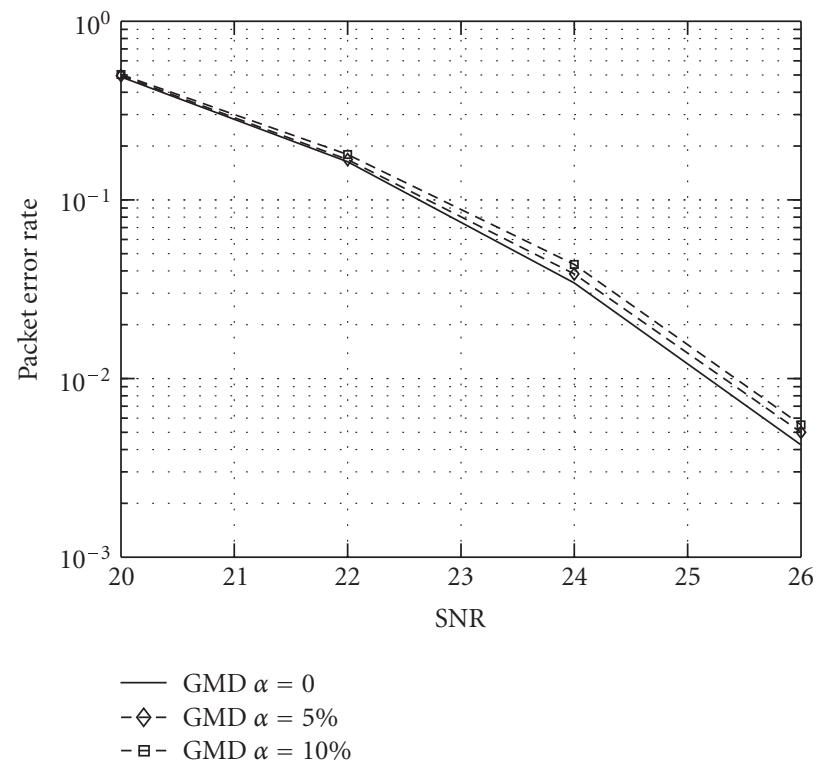

FIGURE 8: Performance comparison of the proposed closed-loop schemes for uncorrelated channels under channel mismatches with different error parameters in the TDD mode for GMD.

Finally, we consider the TDD mode. Figures 8 and 9 show that our closed-loop schemes are quite robust against the mismatches between the channel matrices obtained at the transmitter and the receiver. Our closed-loop schemes suffer from less than $0.2 \mathrm{~dB}$ loss at PER $=0.1$ even when the error parameter is as high as $\alpha=0.1$.

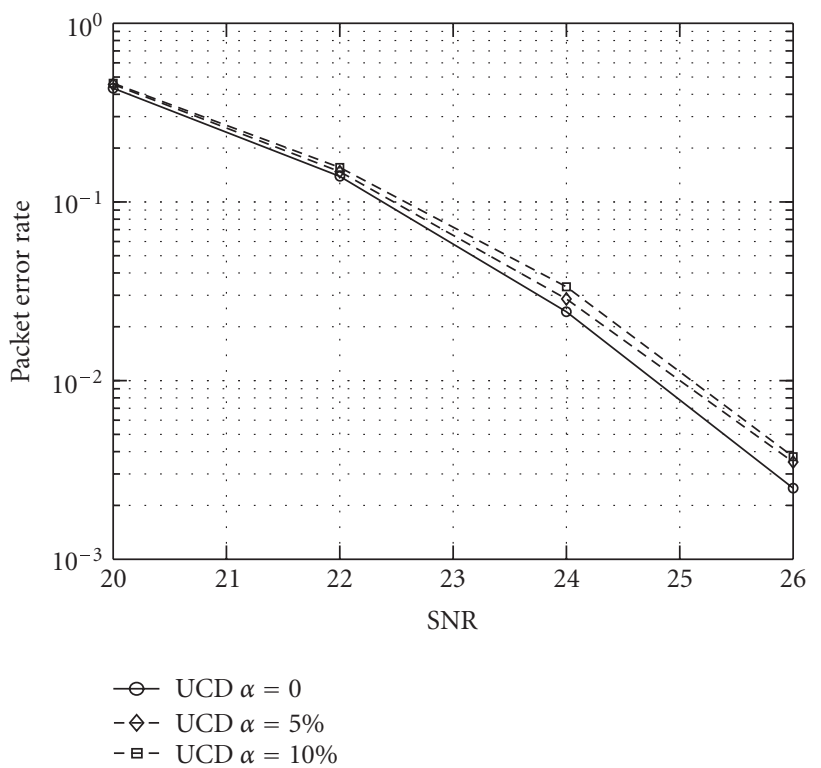

Figure 9: Performance comparison of the proposed closed-loop schemes for uncorrelated channels under channel mismatches with different error parameters in the TDD mode for UCD.

\section{CONCLUSIONS}

We have presented simple and efficient closed-loop designs for MIMO-OFDM-based WLANs as a promising technology for the next-generation wireless LAN communications. By combining the recent GMD and UCD transceiver designs and the horizontal encoding architecture, we can achieve multi- $\mathrm{dB}$ improvement over the closed-loop SVDbased schemes and the open-loop MMSE V-BLAST architecture. The advantage of our schemes is even more prominent when the fading channels are spatially correlated. We have also proposed an efficient algorithm for the quantization of $2 \times 2$ unitary precoders. Using only a 6-bit vector quantization at each data subcarrier, the system can achieve performance very close to the perfect precoder feedback, which represents a very moderate feedback overhead in the explicit feedback mode. In the TDD mode, when the channel reciprocity mechanism is available, we can modify our closedloop designs to be robust against the mismatches between the forward channel and reverse channel. The extensive numerical experiments validate the superior performance of the proposed schemes. Finally, we remark that, although our discussions focus on the $2 \times 2$ system, our schemes can be readily extended to the case of more transmit and more receive antennas.

\section{APPENDIX}

Let $\mathbf{v}_{n} \triangleq\left[\begin{array}{lll}v_{n 1} & v_{n 2} & v_{n 3}\end{array}\right]^{T}=\left[\begin{array}{lll}\cos \theta & \sin \theta \cos \phi & \sin \theta \sin \phi\end{array}\right]^{T}$, and $\tilde{\mathbf{v}}_{i} \triangleq\left[\begin{array}{lll}\tilde{v}_{i 1} & \tilde{v}_{i 2} & \tilde{v}_{i 3}\end{array}\right]^{T}$. The optimization problem in (19) becomes

$$
\min \sum_{\mathbf{v}_{n} \in S_{i}}\left\|\mathbf{v}_{n}-\widetilde{\mathbf{v}}_{i}\right\|^{2} \quad \text { s.t. }\left\|\tilde{\mathbf{v}}_{i}\right\|^{2}=1
$$


The Lagrangian corresponding to the constrained optimization problem is

$$
\mathcal{L}\left(\widetilde{\mathbf{v}}_{i}, \lambda\right)=\sum_{\mathbf{v}_{n} \in S_{i}} \sum_{l=1}^{3}\left(v_{n l}-\tilde{v}_{i l}\right)^{2}+\lambda\left(\sum_{l=1}^{3} \tilde{v}_{i l}^{2}-1\right) .
$$

From the first derivative conditions:

$$
\frac{\partial \mathcal{L}\left(\tilde{\mathbf{v}}_{i}, \lambda\right)}{\partial \tilde{v}_{i l}}=0, \quad l=1,2,3,
$$

and $\left\|\tilde{\mathbf{v}}_{i}\right\|=1$, we have

$$
\tilde{v}_{i l}=\frac{\sum_{\mathbf{v}_{n} \in S_{i}} v_{n l}}{\sqrt{\sum_{l=1}^{3}\left[\sum_{\mathbf{v}_{n} \in S_{i}} v_{n l}\right]^{2}}}, \quad l=1,2,3 .
$$

Define $\overline{\mathbf{v}}_{i}=\left[\begin{array}{lll}\bar{v}_{i 1} & \bar{v}_{i 2} & \bar{v}_{i 3}\end{array}\right]^{T}$, where $\bar{v}_{i l}=\sum_{\mathbf{v}_{n} \in S_{i}} v_{n l} / \sum_{\mathbf{v}_{n} \in S_{i}} 1$, $l=1,2,3$. Then we obtain

$$
\widetilde{\mathbf{v}}_{i}=\frac{\overline{\mathbf{v}}_{i}}{\left\|\overline{\mathbf{v}}_{i}\right\|} .
$$

\section{ACKNOWLEDGMENTS}

This work was supported in part by the National Science Foundation Grant CCR-0104887 and the work of Xiayu Zheng was also supported in part by the University of Florida Alumni Fellowship.

\section{REFERENCES}

[1] IEEE Standard 802.11a-1999, "Supplement to information technology-telecommunications and information exchange between systems-local and metropolitan area networksspecific requirements-part 11: wireless LAN medium access control (MAC) and physical layer (PHY) specifications: high speed physical layer (PHY) in the 5 GHz band," 1999.

[2] J. Liu and J. Li, "A MIMO system with backward compatibility for OFDM-based WLANs," Eurasip Journal on Applied Signal Processing, vol. 2004, no. 5, pp. 696-706, 2004.

[3] IEEE 802.11-04/0889r6, "TGn Sync proposal technical specification," May 2005.

[4] S. Nanda, R. Walton, J. Ketchum, M. Wallace, and S. Howard, "A high-performance MIMO OFDM wireless LAN," IEEE Communications Magazine, vol. 43, no. 2, pp. 101-109, 2005.

[5] P. Xia, S. Zhou, and G. B. Giannakis, "Adaptive MIMO-OFDM based on partial channel state information," IEEE Transactions on Signal Processing, vol. 52, no. 1, pp. 202-213, 2004.

[6] H. Sampath and A. J. Paulraj, "Joint transmit and receive optimization for high data rate wireless communication using multiple antennas," in Proceedings of the 33rd Asilomar Conference on Signals, Systems and Computers (ACSSC '99), vol. 1, pp. 215-219, Pacific Grove, Calif, USA, October 1999.

[7] ETSI, "Broadband radio access networks (BRAN); HIPERLAN type 2: physical (PHY) layer," ETSI TS 101475 V1.2.2, February 2005.

[8] Y. Jiang, J. Li, and W. W. Hager, "Joint transceiver design for MIMO communications using geometric mean decomposition," IEEE Transactions on Signal Processing, vol. 53, no. 10, pp. 3791-3803, 2005.

[9] Y. Jiang, J. Li, and W. W. Hager, "Uniform channel decomposition for MIMO communications," IEEE Transactions on Signal Processing, vol. 53, no. 11, pp. 4283-4294, 2005.
[10] J.-K. Zhang, A. Kavčić, and K. M. Wong, "Equal-diagonal QR decomposition and its application to precoder design for successive-cancellation detection," IEEE Transactions on Information Theory, vol. 51, no. 1, pp. 154-172, 2005.

[11] C.-N. Chuah, D. N. C. Tse, J. M. Kahn, and R. A. Valenzuela, "Capacity scaling in MIMO wireless systems under correlated fading," IEEE Transactions on Information Theory, vol. 48, no. 3, pp. 637-650, 2002.

[12] X. Li, H. Huang, G. J. Foschini, and R. A. Valenzuela, "Effects of iterative detection and decoding on the performance of BLAST," in Proceedings of IEEE Global Telecommunications Conference (GLOBECOM '00), vol. 2, pp. 1061-1066, San Francisco, Calif, USA, November-December 2000.

[13] L. Zheng and D. N. C. Tse, "Diversity and multiplexing: a fundamental tradeoff in multiple-antenna channels," IEEE Transactions on Information Theory, vol. 49, no. 5, pp. 1073-1096, 2003.

[14] B. Hassibi, "A fast square-root implementation for BLAST," in Proceedings of the 34th Asilomar Conference on Signals, Systems and Computers (ACSSC '00), vol. 2, pp. 1255-1259, Pacific Grove, Calif, USA, October-November 2000.

[15] Y. Linde, A. Buzo, and R. Gray, "An algorithm for vector quantizer design," IEEE Transactions on Communications, vol. 28, no. 1, pp. 84-95, 1980.

[16] G. Lebrun, J. Gao, and M. Faulkner, "MIMO transmission over a time-varying channel using SVD," IEEE Transactions on Wireless Communications, vol. 4, no. 2, pp. 757-764, 2005.

[17] N. Chayat, "Tentative criteria for comparison of modulation methods," document: IEEE P802.11-97/96, September 1997.

[18] G. J. Foschini, G. D. Golden, R. A. Valenzuela, and P. W. Wolniansky, "Simplified processing for high spectral efficiency wireless communication employing multi-element arrays," IEEE Journal on Selected Areas in Communications, vol. 17, no. 11, pp. 1841-1852, 1999.

[19] B. Lu, G. Yue, and X. Wang, "Performance analysis and design optimization of LDPC-coded MIMO OFDM systems," IEEE Transactions on Signal Processing, vol. 52, no. 2, pp. 348-361, 2004.

Xiayu Zheng received the B.S. and M.S. degrees in electrical engineering and information science from the University of Science and Technology of China (USTC), Hefei, China, in 2001 and 2004, respectively. He is currently pursuing the Ph.D. degree with the Department of Electrical and Computer Engineering, University of Florida, Gainesville. His research interests are in the areas of signal processing and wireless communications.

Yi Jiang received the B.S. degree in electrical engineering and information science from the University of Science and Technology of China (USTC), Hefei, China, in 2001. He received the M.S. and Ph.D. degrees from the University of Florida, Gainesville, both in electrical engineering, in 2003 and 2005, respectively. In the Summer of 2005, he was a Research Consultant with Information Science Technologies Inc. (ISTI), Fort

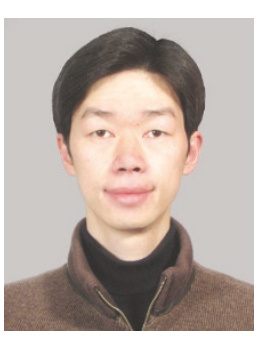
Collins, Colo. He is now a Postdoc with the University of Colorado, Boulder. His research interests are in the areas of signal processing, wireless communications, and information theory.

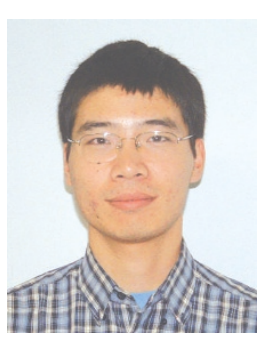


Jian Li received the M.S. and Ph.D. degrees in electrical engineering from The Ohio State University, Columbus, in 1987 and 1991, respectively. From July 1991 to June 1993, she was an Assistant Professor with the Department of Electrical Engineering, University of Kentucky, Lexington. Since August 1993, she has been with the Department of Electrical and Computer Engineering, University of Florida, Gainesville,

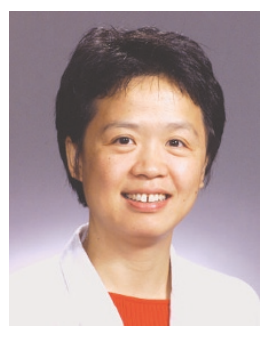
where she is currently a Professor. Her current research interests include spectral estimation, statistical and array signal processing, and their applications. She is a Fellow of IEEE and a Fellow of IEE. She received the 1994 National Science Foundation Young Investigator Award and the 1996 Office of Naval Research Young Investigator Award. She has been a Member of the Editorial Board of Signal Processing, a publication of the European Association for Signal Processing (EURASIP), since 2005. She is presently a Member of two of the IEEE Signal Processing Society technical committees: the Signal Processing Theory and Methods (SPTM) Technical Committee and the Sensor Array and Multichannel (SAM) Technical Committee. 